\title{
Air Conditioning Compressor Air Leak Detection by Image Processing Techniques for Industrial Applications
}

\author{
Kritsada Pookongchai ${ }^{1, a}$, Prasit Nakornrat ${ }^{2, b}$, Bongkoj Sookananta ${ }^{2, c}$, Panhathai Buasri ${ }^{1, d}$ \\ Green Energy Development Research Laboratory, Department of Electrical Engineering, Faculty of Engineering, Khon Kaen University, \\ Khon Kaen, 40002, Thailand \\ ${ }^{2}$ Department of Electrical and Electronic Engineering, Faculty of Engineering, Ubonratchathani University, Ubonratchathani, 34190, \\ Thailand
}

\begin{abstract}
This paper presents method to detect air leakage of an air conditioning compressor using image processing techniques. Quality of air conditioning compressor should not have air leakage. To test an air conditioning compressor leak, air is pumped into a compressor and then submerged into the water tank. If air bubble occurs at surface of the air conditioning compressor, that leakage compressor must be returned for maintenance. In this work a new method to detect leakage and search leakage point with high accuracy, fast, and precise processes was proposed. In a preprocessing procedure to detect the air bubbles, threshold and median filter techniques have been used. Connected component labeling technique is used to detect the air bubbles while blob analysis is searching technique to analyze group of the air bubbles in sequential images. The experiments are tested with proposed algorithm to determine the leakage point of an air conditioning compressor. The location of the leakage point was presented as coordinated point. The results demonstrated that leakage point during process could be accurately detected. The estimation point had error less than $5 \%$ compared to the real leakage point.
\end{abstract}

\section{Introduction}

The air conditioning compressor manufacturing has four main subprocesses(trim a metal chassis, install electrical and mechanical into a compressor, chassis assembly and quality inspection). Air conditioning compressor was a major part in air conditioning and refrigerator manufacturers. During the compressor manufacturing process, incomplete welds process may cause very small leaks that hard to be detected by human. Before shipping the air conditioning compressor out of the manufacture the assembled compressor will be inspected to check the air conditioning compressor quality by leakage detection and searching for leakage points. Leakage detection by human causing error therefore computer vision is implemented to reduce costs of quality inspection compressors and increases the accuracy, rapid and reliability of the quality inspection process. A. Rosenfeld and J. L. Pfaltz were proposed connected component labeling search for connected pixels of object in digital image [1]. W. Burger and M. J. Burge were proposed bounding box and centroid calculation of binary regions called blob analysis [2]. In this work, the connected component labeling and blob analysis were chosen. The inspection method can be applied to other closed tank products that air bubbles leakage is needed to be reduced and measured.

\section{Air Conditioning Compressor Inspection Test Setup}

In this paper, we propose a new method to detect leakage and search leakage point is to improve the accuracy, fast and precise inspection process, better than human inspection. If compressed air in a compressor so that leakage was occurred. Air bubbles escaped from leakage point and float over to surface the water due to air pressure within a compressor.

In a compressor inspection test setup, the light source was installed above so that air bubbles obviously are seen in white color. All curtain scenes are black to prevent external light noise [3], as shown Fig. 1.

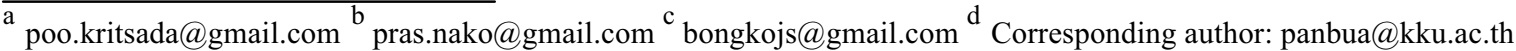



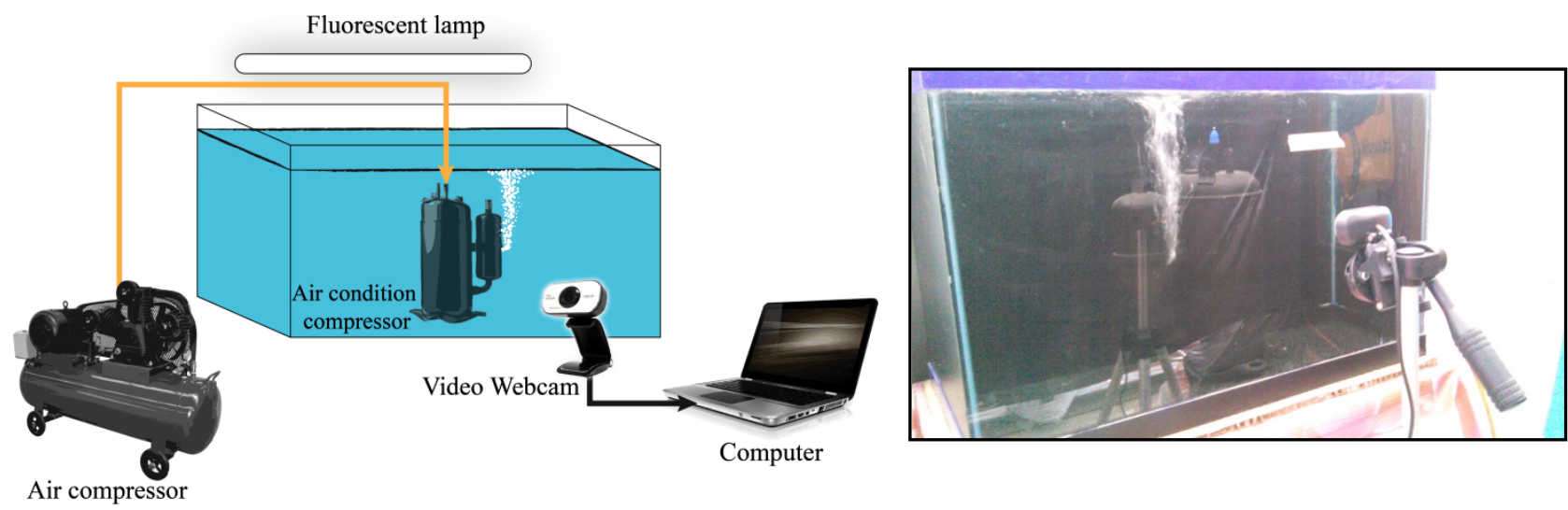

Figure 1. Air conditioning compressor inspection test setup

In figure. 1, a schematic diagram of the air conditioning compressor inspection test setup is presented. A compressor is compressed air with under pressure of 5 bars from the air pump. And then submerge into the water transparent glass tank and fix the test compressor at middle position of the glass tank. Sequential images are taken by a video webcam (OKER HD model 386). A computer with the image processing algorithm was used to record and shows the inspection result in live video.

\section{Image Processing Algorithm}

Image sequences acquired from a video camera is in bitmap files and they were processed before the initial image processing to detect leakage by connected component labeling technique and search leakage point by blob analysis technique.

\subsection{Connected Component Labeling Technique}

Connected component labeling is image processing technique used to detect connected region in binary digital images [4,5]. Connectivity is determined by the medium; image graphs, for example, can be 4-connected or 8-connected [2].

\subsection{Blob Analysis Technique}

Blob analysis is image processing technique used to detect blobs in images and make selected measurements of those blobs shown in Fig. 2. Center of mass (or center of gravity or centroid) of a blob (xc, yc) is calculated as

$$
x_{c}=\frac{1}{N} \sum_{i-1}^{N} x_{i}, \quad y_{c}=\frac{1}{N} \sum_{i=1}^{N} y_{i}
$$

where $\mathrm{N}$ is the number of pixels in the BLOB and xi and $\mathrm{yi}$ are the $\mathrm{x}$ and $\mathrm{y}$ coordinates of the $\mathrm{N}$ pixels, respectively.

Bounding box of a blob is the minimum rectangle which contains the blob. It is defined by going through all pixels for a blob and finding the four pixels with the minimum $x$-value, maximum $x$-value, minimum $y$-value and maximum y-value, respectively. From these values the width of the bounding box is given as xmax - xmin and the height as ymax - ymin. A bounding box can be used as region of interest. Center of the bounding box $(\mathrm{xbb}, \mathrm{ybb})$ is calculated as

$$
\begin{aligned}
& x_{b b}=x_{\text {min }}+\frac{x_{\text {max }}-x_{\text {min }}}{2}= \\
& x_{\text {min }}+\frac{x_{\text {max }}}{2}-\frac{x_{\text {min }}}{2}=\frac{x_{\text {min }}+x_{\text {max }}}{2} \\
& y_{b b}=y_{\text {min }}+\frac{y_{\text {max }}-y_{\text {min }}}{2}= \\
& y_{\text {min }}+\frac{y_{\text {max }}}{2}-\frac{y_{\text {min }}}{2}=\frac{y_{\text {min }}+y_{\text {max }}}{2}
\end{aligned}
$$

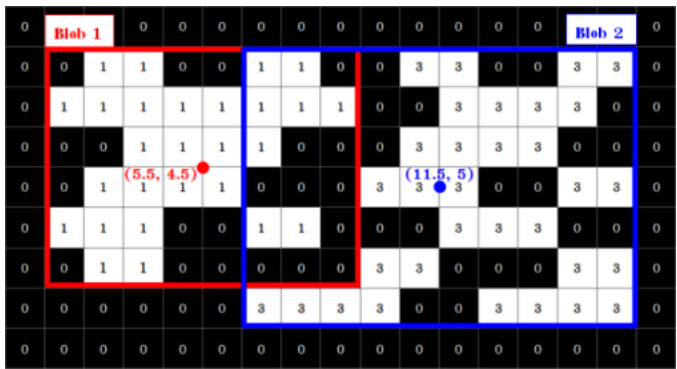

Figure 2. The centroid and bounding box of a blob

Generally, the propose algorithm can be summarized as follows:

1. Initialize the threshold for white detection and number of initial frame.

2. Color images are acquired from a video camera converted to binary images to separate interest objects from a background. Binary images are filtered a noise by a median filter.

3. Use connected component labeling technique (8connectivity) to detect a group of white pixels which is air bubbles of interest, we implement the two-pass algorithm iterates through binary data in an image. Use blob analysis technique to detect blobs in an image, we will get a centroid of blob and value of bounding box. 
4. Compare yc of a blob with previous frame and make a marker at this blob if yc has a value greater than a previous frame. Display the result.

5. Check for the end frame.

The above procedure can also be described by the flowchart shown in Fig. 3.

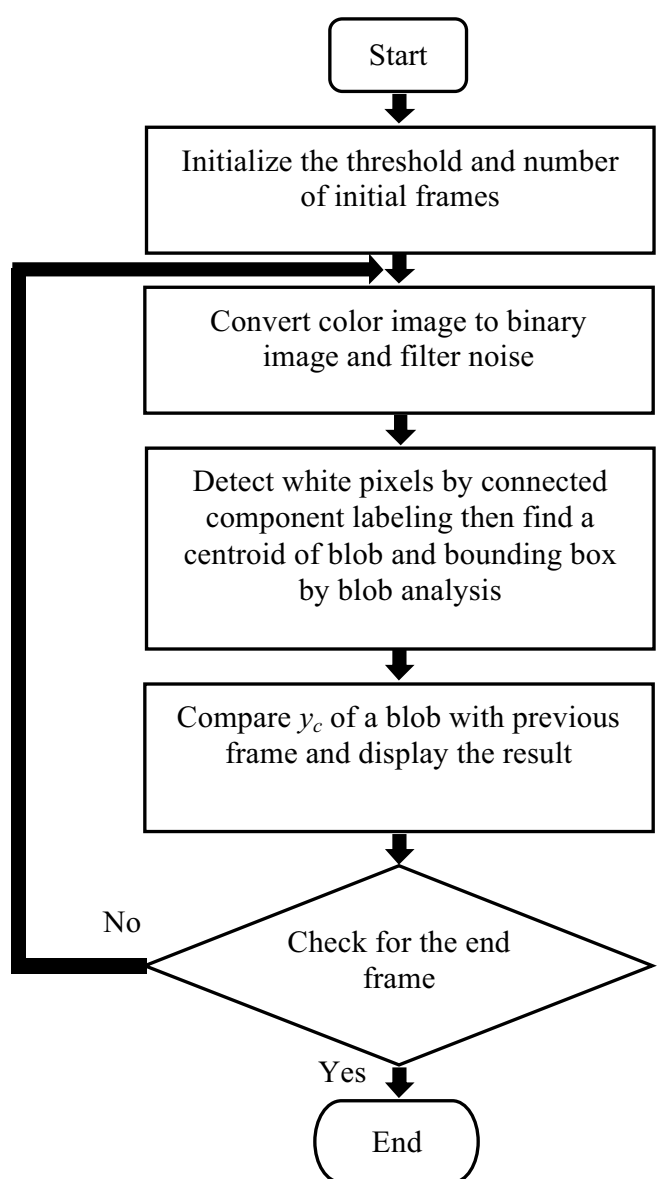

Figure 3. The detailed procedure of a proposed algorithm

\section{Experimental Result}

Figure 3 shows a color image from a video camera and a preprocessed image. The experiments were performed using a computer programming compatible with laptop CPU Intel(R) core(TM) 2 Duo P7350. The original image size is $800 \times 600$ pixels.

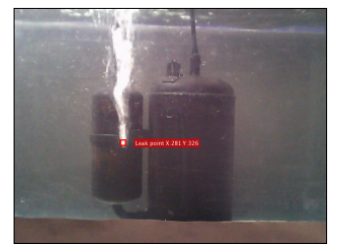

(a)

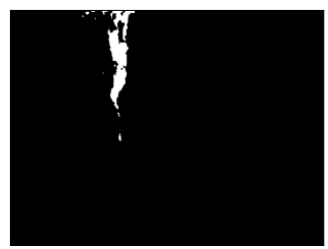

(b)
Figure 4. Preprocessing by convert color image (a) to binary image (b) and filter a noise

Figure 5(a) - 5(c) shows the red rectangle marker of leakage point after processing in difference frame in image sequences to detect the first point to search by connected component labeling technique and blob analysis technique respectively.

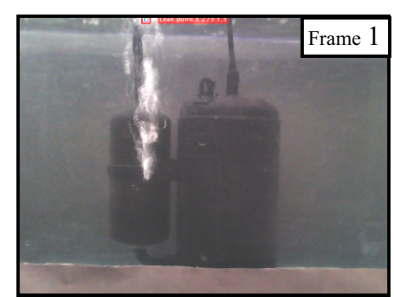

(a)

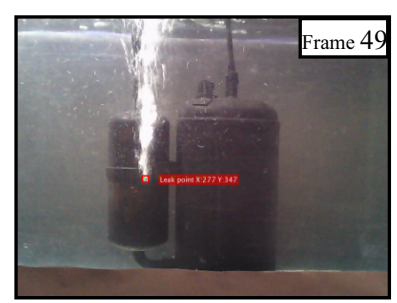

(c)

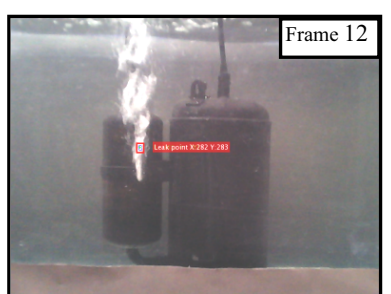

(b)

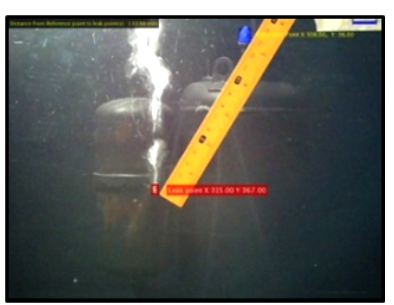

(d)
Figure 5(d) shows the result of the estimation leakage point and measuring point. The accuracy is $95 \%$ by distance from reference point to identify leakage point.

\section{Conclusion}

A new method to detect leakage area and search for leakage point with high accuracy, fast and reliable inspection process was proposed. The method can be used to detect air bubbles escape from an air conditioning compressor. A simple image processing algorithm consists of connected component labeling technique and blob analysis technique. The results demonstrated that the proposed method can identify the leakage in an air conditioning compressor effectively within accuracy of $95 \%$. The proposed method provides benefits for industrial application such as cost reduction, reliability improvement of manufacturing process and value treatment of products.

\section{Acknowledgement}

Authors would like to thank Department of Electrical Engineering, Faculty of Engineering, Khon Kaen University for partially support.

\section{References}

1. A. Rosenfeld, P. Pfaltz: Sequential Operations in Digital Picture Processing. Journal of the ACM Vol.12 (1966),p. 471

2. W. Burger, M. J. Burge: Principles of Digital Image Processing Core Algorithms. Springer-Verlag, London, UK (2009), in press.

3. S. Bongkoj, P. Mongkol, K. Jittiwat and S. Napawan: A study of tank leakage inspection using image 
processing. The $35^{\text {th }}$ Electrical Engineering Conference Vol.2 (2012),p. 836

4. H. Samet, M. Tamminen: Efficient Component Labeling of Images of Arbitrary Dimension Represented by Linear Bintrees. IEEE Transactions on Pattern Analysis and Machine Intelligence Vol.10 (1988),p. 579.

5. M. B. Dillencourt, H. Samet and M. Tamminen: $A$ General Approach to Connected-Component Labeling for Arbitrary Image Representations. Journal of the ACM Vol.39 (1992), p. 253 